\title{
Relation entre taux de couverture du sol et biomasse de résidus de cultures: une simplification prédictive est envisageable
}

\author{
Pascal Thiebeau* \\ INRA, UMR 614 Fractionnement des Agro Ressources et Environnement, 2, Esplanade R. Garros, 51100 Reims, France
}

\begin{abstract}
Résumé - En système d'agriculture de conservation, les résidus de cultures ne sont plus brassés dans un volume de sol labouré mais maintenus à la surface des sols ou partiellement enfouis. Leur présence contribue à la durabilité de ces systèmes par le maintien des équilibres agronomiques et environnementaux qu'ils induisent. Dans ce contexte, la connaissance de l'évolution de leur quantité à la surface d'un sol, de la récolte au semis de la culture suivante, devient un enjeu important de gestion des sols. Plusieurs auteurs ont établi une relation entre le taux de couverture du sol (TC) et la biomasse, propre à chaque espèce végétale, à partir d'un formalisme développé par Grégory (1982). Les objectifs de ce travail sont de tester sa validité dans le contexte pédoclimatique de la région Grand-Est (France), après récolte et au cours du processus de décomposition des résidus. Ce travail a été réalisé sur des exploitations pratiquant l'agriculture de conservation depuis plusieurs années. Les résidus ont été broyés lors de la récolte ou d'une opération spécifique. Les données de biomasse ont été collectées à l'aide de cadres de $0,5 \mathrm{~m} \times 0,5 \mathrm{~m}$, tandis que la couverture du sol a été étudiée à l'aide de photographies numériques. Le taux de couverture du sol a été calculé à l'aide d'un logiciel d'analyse d'images. Ce travail compare également deux périodes de mesure : en sortie d'hiver, plusieurs mois après la récolte, et juste après la récolte estivale. Les résultats montrent une très bonne correspondance entre le TC et la biomasse mesurée au sol, toutes espèces et période de mesures confondues, moyennant l'ajout d'un paramètre au formalisme de Grégory (1982). L'intégration de la variabilité spatiale au sol permet de proposer l'emploi d'un ajustement linéaire unique qui simplifie la prédiction du TC du sol ou «la prédiction» de la quantité de biomasse.
\end{abstract}

Mots clés : agriculture de conservation / variabilité spatiale / décomposition / analyse d'image numérique / modélisation

\begin{abstract}
Relationship between soil cover rate and biomass of crop residues: a predictive simplification is possible. In a conservation agriculture system, crop residues are no longer mixed in a volume of ploughed soil, but maintained on the surface or partially buried. Their presence contributes to the sustainability of these systems by maintaining the agronomic and environmental balances. In this context, knowledge of the evolution of their quantity on the soil surface, from harvest to sowing of the next crop, becomes an important stake of soil management. Several authors have established a relationship between the soil cover ratio and biomass, specific to each plant species, from an identical formalism developed by Gregory (1982). The objectives of this work are to test its use in the pedoclimatic context of the Grand-Est region (France), after harvesting and during the residue degradation process, on several crops. This work has been carried out on farms practicing conservation agriculture for several years. The residues were ground during the harvest or a subsequent specific operation. Biomass data were collected using $0.5 \mathrm{~m} \times 0.5 \mathrm{~m}$ frames, while ground cover were studied using digital photographs. The cover rates were calculated using an image analysis software. This work also compares two measurement periods: at the end of winter, several months after the harvest, and right after the summer harvest. The results show a very good correspondence between the soil cover ratio and the biomass measured on the ground, for all species and measurement periods, by adding a parameter to the Gregory (1982) formalism. The integration of spatial variability on the ground makes it possible to propose the use of a single linear adjustment that simplifies the prediction of soil cover ratio or the amount of biomass.
\end{abstract}

Keywords: conservation agriculture / spatial variability / decomposition / digital image analysis / modelling

\footnotetext{
*Auteur de correspondance : pascal.thiebeau@inra.fr
} 
Tableau 1. Coefficients d'ajustement de propension à la couverture du sol de différents résidus de cultures issus de la littérature. Table 1. Propensity adjustment factors for soil cover of different crop residues from the literature.

\begin{tabular}{|c|c|c|c|c|}
\hline Auteur(s) & Type de résidus & Coef. $a^{a}\left(m^{-2} \cdot g\right.$ MST $)$ & Validité (t.ha $\left.{ }^{-1}\right)$ & $\mathrm{R}^{2}$ \\
\hline \multirow[t]{2}{*}{ Gregory (1982) } & Blé & 0,00500 & $0-10$ & / \\
\hline & Maïs & 0,00400 & $0-10$ & / \\
\hline Dickey et al. (1985) & Maïs & 0,00086 & $0-5$ & 0,721 \\
\hline Gilley et al. (1986) & Maïs & 0,00114 & $0-14$ & 0,995 \\
\hline \multirow[t]{2}{*}{ Gilley et al. (1991) } & Blé & 0,01100 & $0,25-4$ & 0,997 \\
\hline & Maïs & 0,00155 & $2-10$ & 0,978 \\
\hline Khan et al. (1988) & Paille de blé & 0,00670 & $0-5$ & / \\
\hline \multirow[t]{2}{*}{ Scopel et al. (1999) } & Maïs récolte $(\mathrm{R})$ & 0,00367 & $0-20$ & / \\
\hline & Maïs partiellement décomposé (D) & 0,00271 & $0-20$ & / \\
\hline
\end{tabular}

\footnotetext{
${ }^{\mathrm{a}}$ Coefficient de propension à la couverture du sol.
}

\section{Introduction}

En systèmes d'agriculture de conservation, les agriculteurs substituent au labour annuel des techniques culturales de travail du sol limitées aux premiers centimètres de sol ou réalisent le semis des cultures sans aucune préparation du sol. Les résidus de cultures, ainsi maintenus à la surface des sols, sont considérés comme des atouts (Kassam et al., 2015): la réduction des travaux agricoles engendrée nécessite moins d'énergie fossile, ce qui réduit les émissions de gaz à effet de serre. Ils évitent l'exposition des sols aux risques d'érosion éolien et hydrique (Bruce et al., 1995; Smets et al., 2008; Paustian et al., 2016). L'épaisseur de matériaux qu'ils constituent à la surface des sols réduit l'évaporation de l'eau du sol au bénéfice des cultures en place et de la vie biologique du sol (Scopel et al., 2004 ; Fuchs et Hadas, 2011 ; Ranaivoson et al., 2017; Prestele et al., 2018) et atténue les variations de température du sol (Wilhelm et al., 1986; Bussière et Cellier, 1994). Enfin, ces résidus constituent un stockage temporaire de carbone et d'éléments nutritifs qui seront libérés progressivement au fur et à mesure de leur dégradation (Schomberg et Steiner, 1999; Aziz et al., 2013). Dans cette perspective, si la dynamique de leur dégradation à la surface des sols peut être prédite (Galdos et al., 2010; Thiébeau et Recous, 2017), il reste important de maintenir une couverture du sol suffisante au cours du temps, afin de le protéger des agressions du climat (Dickey et al., 1985 ; Smets et al., 2008). La connaissance de l'évolution du taux de couverture du sol (TC) au cours du temps est donc importante à évaluer pour donner aux agriculteurs la possibilité d'adapter leurs pratiques aux risques encourus et de réduire l'impact de leur activité sur l'environnement.

Gregory (1982), Gilley et al. (1986, 1991), Khan et al. (1988), Scopel et al. (1999) ont étudié la relation entre le taux de couverture du sol et la biomasse de paillis. Ils ont obtenu une relation de la forme:

$$
\mathrm{TC}=1-\exp (-\alpha \times \mathrm{MST}),
$$

avec $a$ : coefficient de propension à la couverture du sol;

MST = matière sèche totale du paillis $\left(\mathrm{g} \cdot \mathrm{m}^{-2}\right)$.

Dans ce travail, les auteurs proposent une courbe de référence par espèce (Tab. 1). Pour parvenir à établir cette relation, Gregory (1982) a déterminé le TC par planimétrie des résidus de culture. Plus récemment, Zhao et al. (2012) proposent d'estimer la couverture du sol à l'aide de capteurs de réflectance et du calcul de ratios et d'indices normalisés. Ces méthodes nécessitent des équipements spécifiques. Une autre possibilité pourrait consister à utiliser des moyens numériques. En effet, dans le cadre de mesures de surfaces foliaires, Boissard et al. (1992) sur culture de blé, Andrieu et al. (1997) sur cultures de betterave et de maïs, ont utilisé cette technologie: ils ont mis en relation la surface foliaire des cultures avec le taux de couverture du sol à partir de photographies. C'est pourquoi les photographies ont été utilisées ici pour calculer le TC du sol.

Les objectifs du travail présenté sont:

- d'étudier la couverture du sol de résidus de cultures en place à l'aide de photographies afin de tester la validité de la relation (1) dans le contexte pédoclimatique de la région Grand-Est (France);

- de tester cette relation à deux périodes de l'année (hiver et été) afin de savoir si l'utilisation d'une seule et même relation est suffisante pour prédire le TC du sol.

\section{Matériels et méthode}

Le travail a été réalisé sur des exploitations agricoles pratiquant les Techniques culturales sans labour (TCSL) depuis 5 à 10 ans. Elles se situent en France, en région GrandEst, sur les départements de la Haute-Marne, de l'Aube et de la Meuse. Les mesures ont été réalisées au cours de l'hiver 20082009 (10 champs), puis de l'été 2009 (10 champs). Les champs de chacune des périodes de mesure sont différents.

Les cultures dont les résidus sont étudiés sont le blé (Triticum aestivum L.), l'escourgeon (Hordeum vulgare subsp. hexastichum L.), la féverole (Vicia faba L.), le maïs grain (Zea mays L.), l'orge de printemps (Hordeum vulgare L.) et le tournesol (Helianthus annuus L.). Ces résidus sont composés des tiges, feuilles, restes d'épis, capitules, rafles, menue paille, etc. Ils sont à l'état broyés, soit par le broyeur incorporé aux machines de récolte, soit à l'aide d'un broyeur de paille à axe horizontal pour les résidus de féverole, maïs et tournesol. 
Dans ces situations, l'agriculteur est intervenu 24 à $48 \mathrm{~h}$ après la récolte. Les résidus sont étudiés tels quels, c'est-à-dire dans la position où ils ont été placés sur le sol au cours des opérations culturales.

Les mesures de biomasse et les photographies sont réalisées en fin d'hiver, avant la mise en place des cultures de printemps, ou 24 à $72 \mathrm{~h}$ après chaque récolte en période estivale. Elles sont réalisées sur un transect perpendiculaire au sens de la récolte, correspondant à une largeur de récolteuse, pour rendre compte des variabilités spatiales du sol et de la répartition des résidus au sol lors des récoltes (Thiébeau et Recous, 2016).

\subsection{Mesures de biomasses et photographiques}

L'unité de prélèvement pour l'estimation de la Matière sèche totale (MST) des résidus est constituée d'un cadre de dimensions intérieures de $0,5 \mathrm{~m} \times 0,5 \mathrm{~m}$. Les prélèvements comprennent plusieurs cadres unitaires, constituant chacun des placettes de prélèvement dont le nombre dépend de la largeur de coupe de la récolteuse utilisée. Il en est prélevé 1 par mètre linéaire de largeur de coupe. Les résidus de chaque cadre sont collectés et identifiés. Ils seront passés en étuve ventilée pendant $48 \mathrm{~h}$ à $80^{\circ} \mathrm{C}$ au laboratoire pour la détermination de leur masse sèche. Dans ce travail, 4 à 6 mesures (répétitions) ont été réalisées par champ.

Les photographies de chaque placette de prélèvement de $0,5 \mathrm{~m} \times 0,5 \mathrm{~m}$ sont réalisées avant la collecte des résidus. L'appareil utilisé est muni d'une cellule photographique de 7,1 mégapixels, d'un correcteur de luminosité automatique et d'un stabilisateur d'image. Chaque image est identifiée (date, lieu, numéro de placette) afin de relier a posteriori la biomasse contenue dans le cadre de prélèvement au TC qui sera établi lors du traitement numérique.

Les images sont analysées à l'aide du logiciel Optimas v.6.51 (Meyer Instruments Inc., Houston, Texas, États-Unis). Elles sont codées en trois couleurs primaires sur 256 niveaux d'intensité dans le rouge, le bleu et le vert. Cela permet d'intégrer des contrastes de couleurs liés à l'humidité des paillis et du sol, une luminosité différente entre photographies, etc. Le logiciel permet à l'opérateur d'adapter la procédure en comparant l'image reconstituée après traitement, à l'image originale (Fig. 1).

\subsection{Relation (taux de couverture du sol - quantité de matière sèche totale de résidus)}

L'analyse des images n'étant pas automatisée, une sélection de résidus a été réalisée pour représenter la diversité des quantités de biomasses présentes au sol et observées au champ (Thiébeau et Recous, 2016). La construction de la relation (TC du sol MST de résidus) est réalisée d'abord par confrontation des valeurs unitaires de TC-MST pour chaque cadre de prélèvement et situation collectée, ce qui permet d'observer la variabilité de répartition des résidus à la surface du sol et celle des TC rencontrés; puis, par confrontation des valeurs moyennes observées par champ.

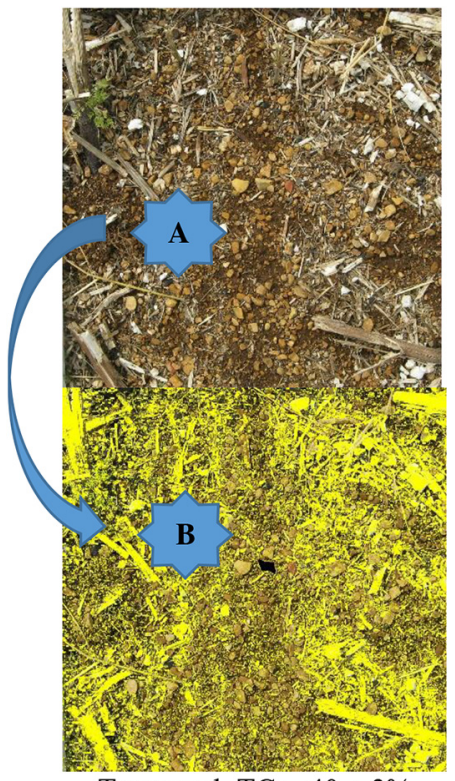

Tournesol, $\mathrm{TC}=40 \pm 3 \%$

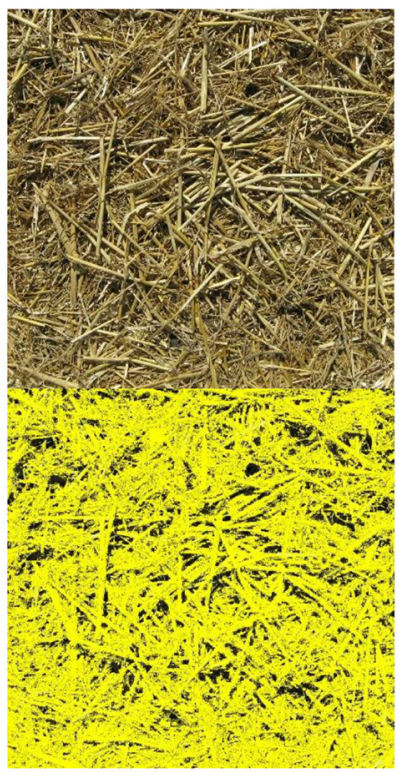

Blé, $\mathrm{TC}=73 \pm 1 \%$
Fig. 1. (A) Exemples d'images brutes de résidus de cultures de tournesol et de blé avant et (B) après leur traitement numérique respectifs : toutes les parties en jaunes sont comptabilisées pour être de la surface couverte par ces résidus.

Fig. 1. (A) Example of raw images of sunflower and wheat crop residues before and $(B)$ after their respective numerical treatment: all the parts in yellow are counted to be of the surface covered by these residues.

\subsection{Traitements statistiques des données}

Toutes les moyennes présentées sont accompagnées de leur erreur d'estimation standardisée (SE) afin de pouvoir être comparées entre elles.

Les ajustements non-linéaires et linéaires ont été réalisés à l'aide du programme statistique de Sigma-Plot 12 (Systat Software Inc., San José, Californie, États-Unis) après validation de la normalité des variables par le test Shapiro-Wilk. Les critères d'évaluation des ajustements sont l'erreur quadratique au modèle (RMSE), sa valeur relative (rRMSE) et le coefficient de détermination $\left(R^{2}\right)$. Ils sont définis comme suit:

$$
R M S E=\sqrt{\frac{1}{n} \cdot \sum_{i=1}^{n}\left(P_{i}-O_{i}\right)^{2}} .
$$

$O$ et $P$ sont respectivement les valeurs observées et prédites, $n$ est le nombre de paires observées-simulées. La RMSE s'exprime dans la même unité que la grandeur physique considérée.

$$
r R M S E=100 \cdot \frac{R M S E}{\bar{O}}
$$

$\bar{O}$ est la moyenne des valeurs observées. Elle est exprimée en $\%$. 
$R^{2}$ est le carré de la corrélation du coefficient de Pearson, entre les valeurs observées et les valeurs simulées. Il décrit la proportion de la variance totale des données observées qui peut être expliquée par le modèle. Il est calculé comme suit:

$$
R^{2}=\left[\frac{\sum_{i=1}^{n}\left(P_{i}-\bar{P}\right) \cdot\left(O_{i}-\bar{O}\right)}{\sigma_{P} \cdot \sigma_{O}}\right] Z,
$$

avec $\bar{P}$ et $\bar{O}$ comme valeurs moyennes respectivement estimées et observées.

Le seuil de signification maximal retenu est $p<0,05$. Pour les intervalles de confiance (IC) et de prédiction (IP) présentés, le seuil de signification retenu est de $95 \%$.

\section{Résultats}

Les données recueillies au cours des deux périodes de mesure se répartissent comme suit: 5 champs en blé, 2 en escourgeon, 1 en orge de printemps, 1 en féverole, 4 en tournesol et 7 en maïs. Au cours de l'hiver 2008/2009, les dix situations retenues comprennent 4 blés, 2 escourgeons, 1 orge, mesurées huit mois après leur récolte et 3 maïs, mesurés cinq mois après leur récolte. Au cours de l'été/automne 2009, les dix situations retenues comprennent 1 blé, 1 féverole, 4 tournesols et 4 maïs. Ces espèces correspondent à l'objectif de travail en situations contrastées, telles que rencontrées dans cette région pour ces systèmes de cultures (Thiébeau, 2019).

\subsection{Comparaison des mesures (TC du sol-MST des résidus) acquises sur chaque placette}

La masse des résidus collectés en fin d'hiver s'étend de 183 à $1218 \mathrm{~g} \mathrm{MST.m}^{-2}$ pour des TC du sol de 22 à $88 \%$. Les résidus collectés en été ont des masses comprises de 282 à 2374 g.m ${ }^{-2}$ pour des TC qui varient de 20 à $100 \%$. Les valeurs de toutes les placettes collectées développent un nuage de points qui montre une cohérence entre le TC du sol estimé et la MST mesurée (Fig. 2). La figure 2A montre que les nuages de points de chacune des périodes de mesure (hiver vs été) se superposent, ce qui autorise leur traitement sans distinction de période de mesure. La figure $2 \mathrm{~B}$ montre que l'ensemble des cultures se confondent, avec une mention particulière pour le maïs dont plusieurs estimations de TC présentent un coefficient maximal de $100 \%$.

La comparaison de ces résultats aux travaux existants (Tab. 1) permet de constater que, dans leur plage de validité, les mesures obtenues sur le blé $\left(0,00200 \mathrm{~m}^{-2}\right.$.g MST en moyenne) dans ce travail décrochent de manière assez nette aux modèles mentionnés (de 0,00500 à $0,01100 \mathrm{~m}^{-2}$.g MST; Fig. 3). Pour le maïs $\left(0,00103 \mathrm{~m}^{-2}\right.$.g MST en moyenne) et le tournesol $\left(0,00189 \mathrm{~m}^{-2}\right.$.g MST en moyenne), les valeurs mesurées au champ se répartissent dans les grandes lignes des modèles retrouvés dans la bibliographie (de 0,00086 à 0,00400 et 0,00102 à $0,00200 \mathrm{~m}^{-2}$.g MST respectivement pour le maïs et le tournesol). Les tentatives d'optimisation des valeurs du coefficient de propension à la couverture du sol «a» de l'équation (1) ne permettent pas d'obtenir un résultat satisfaisant $\left(R^{2}<0,5\right)$. En conservant un modèle non-linéaire, de type

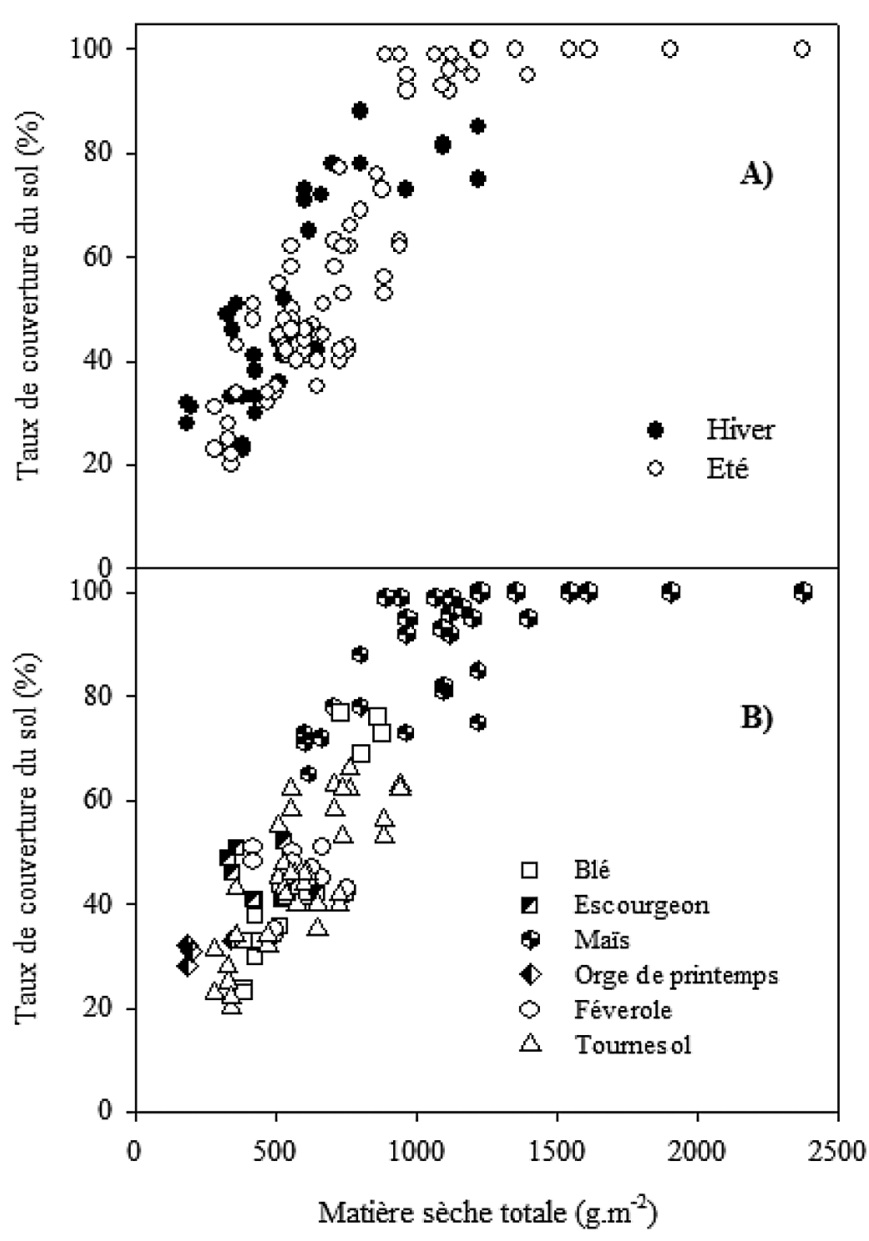

Fig. 2. Relation entre les mesures de matière sèche totale (MST) collectées et le pourcentage de couverture du sol correspondant: (A) Distinction des mesures collectées en hiver de celles collectées en été ; (B) Distinction des mesures collectées par espèces végétales.

Fig. 2. Relationship between the total dry matter (TDM) measurements collected and the corresponding percentage of soil cover: (A) Distinction of the measurements collected in winter from those collected in summer; (B) Distinction of measurements collected by plant species.

exponentiel avec un plateau maximum tel que retenu originellement par Gregory (1982), il faut ajouter un paramètre au modèle (1). Il est alors de la forme :

$$
\mathrm{TC}(\%)=\mathrm{a} \times(1-\exp .(-\mathrm{b} \times \mathrm{MST})),
$$

avec $\mathrm{TC}=$ taux de couverture du sol $(\%) ;$ MST : matière sèche totale de résidus $\left(\mathrm{g} . \mathrm{m}^{-2}\right)$, a et $\mathrm{b}=$ coefficients à optimiser. Les données collectées dans cette étude donnent les valeurs suivantes : $a=148$ et $b=0,0007(\mathrm{RMSE}=11,5 \%$; $\mathrm{RMSE}=$ $\left.19,9 \% ; R^{2}=0,7564 * * *\right)$. Cet ajustement se révèle très hautement significatif $(P<0,001)$. Son tracé, accompagné des intervalles de confiance (traits d'union) et de prédiction (pointillés), est présenté par la figure 4. Toutes les données se trouvent réparties à l'intérieur de ces intervalles, hormis deux données proches d'un TC de $100 \%$. 


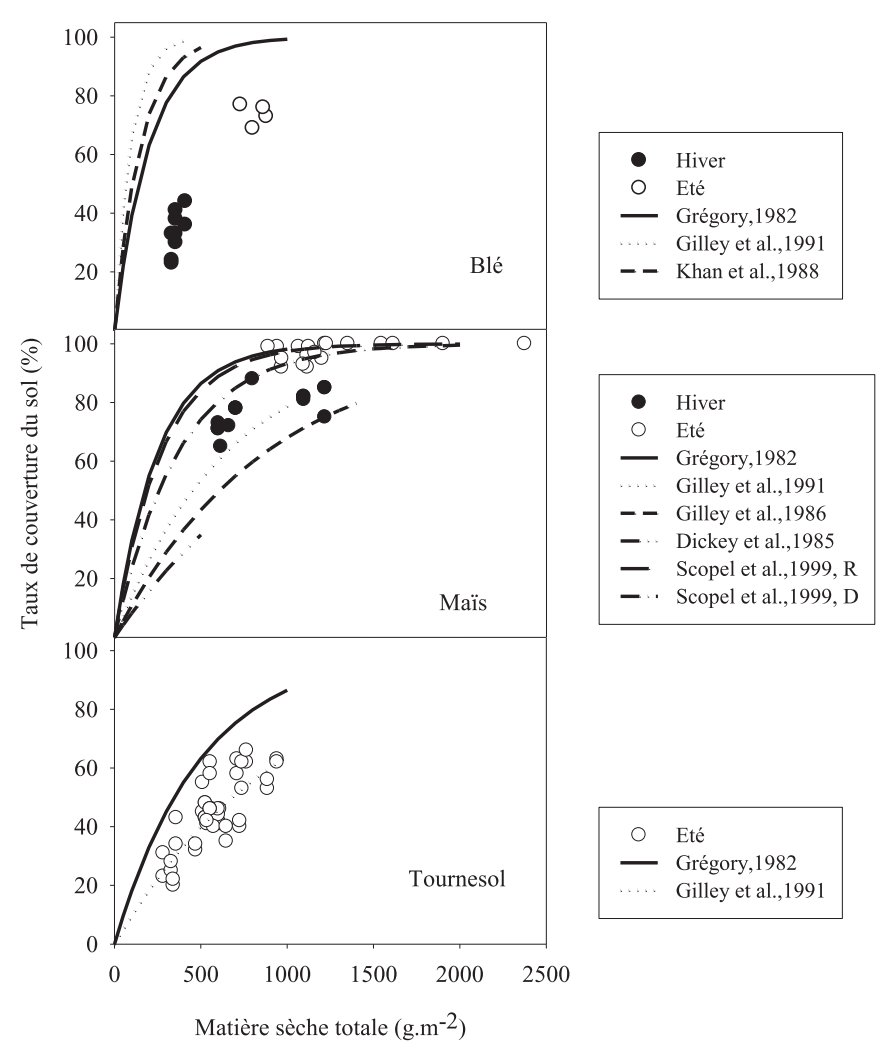

Fig. 3. Comparaison des couples de mesures (MST-TC) collectées pour le blé, le maïs et le tournesol aux ajustements spécifiques à chacune de ces espèces trouvés dans la littérature (Tab. 1). Chaque tracé est limité à son domaine de validité ( $\mathrm{R}$ : récolte; $\mathrm{D}$ : en décomposition).

Fig. 3. Comparison of measurement pairs (MST-TC) collected for wheat, maize and sunflower with the specific adjustments for each of these species found in the literature (Tab. 1). Each line is limited to its area of validity. (R: harvest; $D$ : in decomposition).

\subsection{Comparaison des valeurs moyennes (TC-MST) acquises par champ}

Cette analyse permet d'intégrer la variabilité spatiale de chacune des 20 situations explorées. La masse des résidus collectés et regroupés s'étend alors de $227 \pm 38$ à $1324 \pm 67 \mathrm{~g}$ MST.m ${ }^{-2}$ pour une plage de taux de couverture allant de $28 \pm 3$ à $100 \pm 1 \%$. Le positionnement de ces données sur l'ajustement non-linéaire (2) précédent montre une bonne répartition de ces valeurs moyennes le long du tracé de l'ajustement jusqu'à $1000 \mathrm{~g} \mathrm{MST.m}{ }^{-2}$, quelle que soit l'espèce végétale considérée (Fig. 5A). Au-delà, les points restent à l'intérieur de l'intervalle de prédiction mais s'écartent de l'intervalle de confiance de l'ajustement. Une meilleure prédiction de ces mesures peut être obtenue en utilisant un ajustement de type linéaire :

$$
\mathrm{TC}(\%)=(\mathrm{a} \times \mathrm{MST})+\mathrm{b},
$$

avec: $a$ et $b$, respectivement la pente et la constante de la droite. Le calcul de l'ajustement libre a pour valeurs $a=0,073$ et $b=6,36$ avec $\operatorname{RMSE}=5,9 \% ; \operatorname{rRMSE}=10,0 \%$; $R^{2}=0,9412^{* * *}$. Ce résultat est très hautement significatif

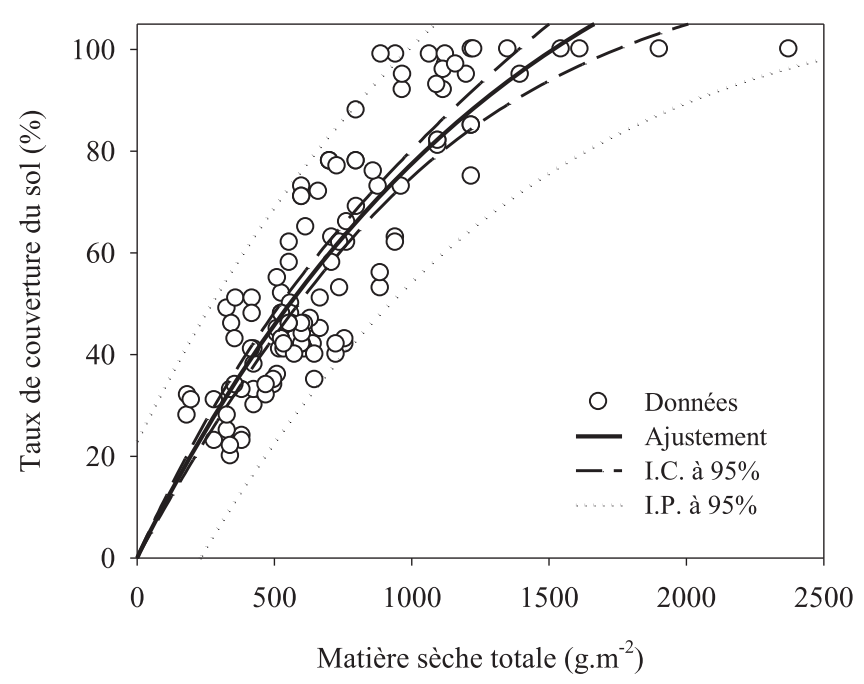

Fig. 4. Relation entre la matière sèche totale (MST) de résidus présente au sol et le taux de couverture (TC) du sol: tracé de l'ajustement non-linéaire, de ses intervalles de confiance et de prédiction à $95 \%$.

Fig. 4. Relationship between Total Dry Matter (MST) of residue on soil and soil coverage (TC): plot of non-linear adjustment, 95\% confidence and prediction intervals.

$(P<0,001)$. L'erreur d'estimation (RMSE) inférieure à $6 \%$ et la rRMSE de $10 \%$ sont de bons résultats. Son tracé, accompagné des intervalles de confiance (traits d'union) et de prédiction (pointillés), est présenté par la figure 5B. L'ensemble des données se trouve réparti à l'intérieur de ces intervalles. Dans la situation d'un ajustement forcé par l'origine, «a» présente une valeur de 0,080 $(\mathrm{RMSE}=6,5 \%$; rRMSE $\left.=11,0 \% ; R^{2}=0,9299, P<0,001\right)$.

Les valeurs inverses de ces fonctions pour MST $=f(\mathrm{TC})$ sont les suivantes :

Ajustement libre : $\mathrm{a}=12,9$ et $\mathrm{b}=-39,7$ avec RMSE $=79,2 \mathrm{~g}$ MST $;$ RMSE $=10,9 \% ; R^{2}=0,9412(P<0,001)$;

Ajustement forcé par l'origine: $\mathrm{a}=12,4$ avec RMSE $=$ $80,6 \mathrm{~g}$ MST; $\mathrm{rRMSE}=11,1 \% ; R^{2}=0,9390(P<0,001)$.

\section{Discussion}

L'étendue des masses de résidus couverte par cette étude $\left(227 \pm 38\right.$ à $\left.1324 \pm 67 \mathrm{~g} \mathrm{MST}^{-2} \mathrm{~m}^{-2}\right)$ est représentative de celles reportées par Thiébeau et Recous (2016): $267 \pm 23$ à $1370 \pm 140 \mathrm{~g} \mathrm{MST}^{-2}{ }^{-2}$; ce qui correspond à la sélection de situations recherchées pour établir la relation (TC du sol MST).

La confrontation des mesures de MST aux TC du sol montre qu'il est possible de proposer un ajustement. Plusieurs auteurs se sont accordés à retenir l'ajustement non-linéaire proposé par Gregory (1982), dont les coefficients « $a$ » sont spécifiques à chaque espèce végétale étudiée. Cependant, au fils de leurs travaux, Gilley et al. $(1986,1991)$ ont proposé des coefficients différents pour une même espèce (Tab. 1). La comparaison de leurs résultats aux mesures réalisées pour trois espèces de ce travail montre que plusieurs mesures s'écartent significativement des TC prédits par modélisation (Fig. 3) et qu'une diversité de situations peut être rencontrée selon les 


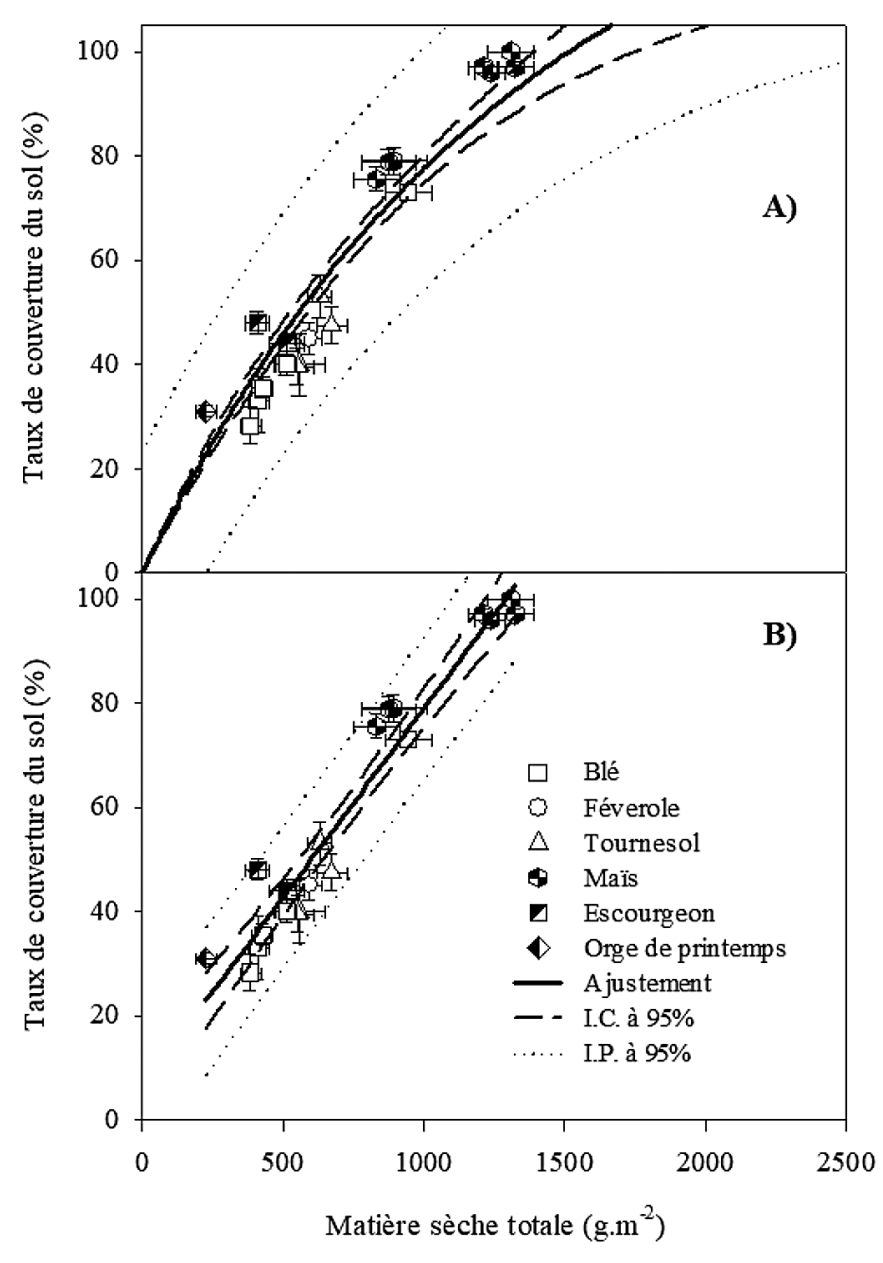

Fig. 5. Comparaison des couples de données (MST-TC) moyens par champ, accompagnés de leur erreur standard, aux ajustements (A) non-linéaire et (B) linéaire établis, avec identification des espèces.

Fig. 5. Comparison of the mean data pairs (MST-TC) by field, accompanied by their standard error, with established (A) non-linear and $(B)$ linear adjustments, and species identification.

conditions expérimentales. Plusieurs hypothèses peuvent être émises pour justifier ces différences :

- la composition biochimique des différents organes des plantes induit des vitesses de décomposition différentes. Ainsi, Scopel et al. (1999) stipulent que ce sont surtout les feuilles et les parties médullaires des tiges qui se décomposent le plus rapidement. Viennent ensuite les parties externes des tiges, plus riches en lignine. La succession de cultures, dont les proportions et compositions diffèrent entre espèces, induisent des vitesses de dégradation hétérogènes au cours de la rotation des cultures et un prolongement de la couverture du sol dans le temps. Par ailleurs, Scopel et al. (1999) ont également démontré que le pouvoir couvrant des organes les plus longs à se dégrader était différent de celui des premiers dégradés, ce qui peut justifier, dans cette étude réalisée en situations réelles rencontrées au champ par les agriculteurs, qu'une variabilité de couverture du sol soit observée pour une même espèce;

- une autre hypothèse réside dans l'ancienneté de mise en œuvre des TCSL sur les exploitations support de ce travail.
Cela a probablement conduit à mesurer des quantités de biomasses supérieures à celles rencontrées en situations avec labours annuels. En effet, comme expliqué précédemment et montré par Thiébeau et Recous (2017), la dégradation des résidus de cultures maintenus à la surface des sols se prolonge sur plusieurs années. En conséquence, le positionnement successif de couches de résidus dont les vitesses de dégradation diffèrent selon les conditions agroenvironnementales, peut avoir augmenté les biomasses mesurées et induit une diminution des coefficients « $a$ » obtenus. Il en résulte, comme l'ont montré Steiner et al. (1999), une variabilité assez conséquente d'estimation du TC selon la MST présente.

L'absence d'écart entre les mesures TC-MST établies en fin d'hiver, avec des résidus de cultures en cours de décomposition, et en été, dès la récolte, constitue un résultat qui conforte les travaux de Steiner et al. (2000) réalisés à partir de résidus de blé et d'avoine, ainsi que ceux de Scopel et al. (1999) sur maïs. À l'image des conclusions de Zhao et al. (2012), ces résultats montrent combien il est délicat de vouloir ajuster une courbe de couverture du sol par espèce végétale pour décrire les situations de biomasse rencontrées en TCSL. En conséquence, cela autorise l'utilisation d'un seul et même ajustement pour construire la relation (TC-MST).

L'ajustement unique proposé dans ce travail reprend la racine de l'équation de Gregory (1982), pour y ajouter un paramètre. Sa qualité de prédiction est bonne $\left(R^{2}>0,75\right)$ avec une estimation d'erreur (rRMSE) proche de $20 \%$. Cette valeur est similaire à ce que Thiébeau (2019) a mentionné dans la relation (épaisseur de résidus-biomasse) en comparant des estimations de biomasse moyennes par champ, et non des valeurs unitaires de placette comme c'est le cas dans la construction de l'ajustement nonlinéaire présenté. Or, dans ce travail, les données moyennes par champ ne permettent pas d'ajuster un modèle non-linéaire. En revanche, le modèle linéaire présente une très bonne qualité de prédiction $\left(R^{2}>0,94\right)$ pour une estimation d'erreur (rRMSE) de $10 \%$. Ainsi, l'estimation de la valeur de biomasse au sol à partir du TC, ou vice-versa, sera plus précise que celle obtenue par la méthode utilisant des mesures d'épaisseur du paillis (rRMSE = $20,2 \%$ ). Ce modèle linéaire est également plus précis que l'ajustement non-linéaire, et plus simple à mettre en œuvre. Il est applicable quelle que soit la culture ou son stade de décomposition. Il montre que la saturation de la couverture du sol $(100 \%)$ est atteinte pour une biomasse de $12,5 \mathrm{t} \mathrm{MST.ha}^{-1}$ (valeur arrondie), ce qui est conséquent lorsque l'on sait qu'une couverture du sol de $20 \%$ permet de réduire de moitié son érosion pluviale (Dickey et al., 1985).

Cette étude complète les connaissances acquises sur la caractérisation des biomasses au sol pour des exploitations mettant en œuvre des TCSL, notamment dans cette région du monde à climat tempéré, où ces systèmes de cultures sont encore peu développés (Prestele et al., 2018). Ces résultats peuvent être intégrés aux modèles sol-plante-atmosphère, comme le modèle STICS (Brisson et al., 2009), pour améliorer le paramétrage existant et mieux simuler le devenir des résidus sous des climats différents (Scopel et al., 2004; Ranaivoson et al., 2017). Des moyens aptes à intégrer des surfaces plus importantes, tels que des photographies aériennes ou satellitaires, pourraient être utilisés pour affiner la relation obtenue et définir l'aire géographique dans laquelle cette simplification est acceptable. 


\section{Conclusion}

Ce travail original permet d'estimer la biomasse de résidus au sol ou son taux de couverture du sol, de la récolte à l'implantation de la culture suivante, à l'aide d'un seul et même ajustement, quelle que soit l'espèce végétale cultivée récoltée à maturité. Le modèle non-linéaire proposé par Gregory (1982) doit être enrichi d'un paramètre pour être employé sur les systèmes mettant en œuvre les TCSL de la région Grand-Est (France). L'ajustement linéaire proposé apparaît plus précis pour déterminer le TC ou la MST au sol. Ce résultat devra être complété par d'autres études afin de définir l'aire géographique dans laquelle cette simplification est acceptable.

Remerciements. L'auteur remercie Messieurs Acker, Ferté, Gublin, Lemey, Martens, Ortillon et Robert, exploitants agricoles qui ont accepté de mettre leurs champs à sa disposition afin que cette étude puisse être conduite. Il remercie Akhtar Iqbal et Francis Millon (INRA Reims) pour leur aide technique lors des prélèvements au champ, ainsi que Pierre Belluomo (INRA Grignon) pour son aide lors des traitements d'images. Il remercie également les relecteurs de la revue qui ont contribué à l'amélioration de cet article. L'agence nationale de la recherche (ANR) est remerciée pour le soutien financier apporté de 2009 à 2013 au programme Systerra, projet PEPITES (Processus écologiques et processus d'innovation technique et sociale en agriculture de conservation).

\section{Références}

Andrieu B, Allirand JM, Jaggard K. 1997. Ground cover and leaf area index of maize and sugar beet crops. Agronomie 17: 315-321. DOI: 10.1051/agro:19970602.

Aziz I, Mahmood T, Islam KR. 2013. Effect of long-term no-till and conventional tillage practices on soil quality. Soil \& Tillage Reseach 13: 28-35. DOI: 10.1016/j.still.2013.03.002.

Boissard P, Pointel JG, Tranchefort J. 1992. Estimation of the ground cover ratio of a wheat canopy using radiometry. International Journal of Remote Sensing 13(9): 1681-1692. DOI: 10.1080/ 01431169208904220.

Brisson N, Launay M, Mary B, Beaudoin N. 2009. Conceptual basis, formalizations and parameterization of the STICS crop model. Ed. Quae c/o Inra Versailles, 297 p.

Bruce RR, Langdale GW, West LT, Miller WP. 1995. Surface soil degradation and soil productivity restoration and maintenance. Soil Science Society of America Journal 59: 654-660. DOI: 10.2136/ sssaj1995.03615995005900030003x.

Bussière F, Cellier P. 1994. Modification of the soil temperature and water content regimes by a crop residue mulch: experiment and modelling. Agricultural \& Forest Meteorology 68: 1-28. DOI: 10.1016/0168-1923(94)90066-3.

Dickey EC, Shelton DP, Jasa PJ, Peterson TR. 1985. Soil erosion from tillage systems used in soybean and corn residues. American Society of Agricultural Engineers 28(4): 1124-1129. DOI: 10.13031/2013.32399.

Fuchs M, Hadas A. 2011. Mulch resistance to water vapor transport. Agricultural Water Management 98: 990-998. DOI: 10.1016/j. agwat.2011.01.008.
Galdos MV, Cerri CC, Cerri CEP, Paustian K, Van Antwerpen R. 2010. Simulation of sugarcane residue decomposition and aboveground growth. Plant \& Soil 326: 243-259. DOI: 10.1007/ s11104-009-0004-3.

Gilley JE, Finkner S, Spomer R, Mielke L. 1986. Runoff and erosion as affected by corn residue: I. Total losses. American Society of Agricultural Engineers 85-2540: 157-164. DOI: 10.13031/ 2013.30119

Gilley JE, Kottwitz ER, Wieman GA. 1991. Roughness coefficients for selected residue materials. Journal of Irrigation and Drainage Engineering 117(4): 503-514. DOI: 10.1061/(ASCE)0733-9437 (1991)117:4(503).

Gregory JM. 1982. Soil cover prediction with various amounts and types of crop residue. American Society of Agricultural Engineers, 1333-1337. DOI: 10.13031/2013.33723.

Kassam A, Friedrich T, Derpsch R, Kienzle J. 2015. Overview of the worldwide spread of conservation agriculture. Field Actions Science Reports 8: 1-11. http://factsreports.revues.org/3966.

Khan M, Monke E, Foster G. 1988. Mulch cover and canopy effect on soil loss. Transactions of the American Society of Agricultural Engineers 31(3): 706-711. DOI: 10.13031/2013.30771.

Paustian K, Lehmann J, Ogle D, Robertson G, Smith P. 2016. Climate-smart soils. Nature 532: 49-57. DOI: 10.1038/ nature17174.

Prestele R, Hirsch A, Davin E, Seneviratne S, Verbug PH. 2018. A spatially explicit representation of conservation agriculture for application in global change studies. Global Change Biology 24: 4038-4053. DOI: $10.1111 /$ gcb.14307.

Ranaivoson L, Naudin K, Ripoche A, Affholder F, Rabeharisoa L, Corbeels M. 2017. Agro-ecological functions of crop residues under conservation agriculture. A review. Agronomy for Sustainable Development 37: 26. DOI: 10.1007/s13593-017-0432-z.

Schomberg HH, Steiner JL. 1999. Nutrient dynamics of crop residues decomposing on a fallow no-till soil surface. Soil Science Society of America Journal 63: 607-613. DOI: 10.2136/ sssaj1999.03615995006300030025x.

Scopel E, Chavez Guerra E, Arreola Tostado JM. 1999. Le semis direct avec paillis de résidus dans l'ouest mexicain : une histoire d'eau? Agriculture et Développement 21: 71-86. https://agritrop. cirad.fr/392540.

Scopel E, Da Silva F, Corbeels M, Affholder F, Maraux F. 2004. Modelling crop residue mulching effects on water use and production of maize under semi-arid and humid tropical conditions. Agronomie 24: 383-395. DOI: 10.1051/agro:2004029.

Smets T, Poesen J, Knapen A. 2008. Spatial scale effects on the effectiveness of organic mulches in reducing soil erosion by water. Earth-Science Reviews 89: 1-12. DOI: 10.1016/j.earsci rev.2008.04.001.

Steiner JL, Schomberg HH, Unger PW, Cresap J. 1999. Crop residue decomposition in no-tillage small-grain fields. Soil Science Society of America Journal 63: 1817-1824. DOI: 10.2136/ sssaj1999.6361817x.

Steiner JL, Schomberg HH, Unger PW, Cresap J. 2000. Biomass and residue cover relationship of fresh and decomposing small grain residue. Soil Science Society of America Journal 64(6): 2109-2114. DOI: $10.2136 /$ sssaj2000.6462109x.

Thiébeau P. 2019. Mesurer l'épaisseur des résidus à la surface d'un sol pour estimer leur biomasse. Cahiers Agricultures 28: 11. DOI: 10.1051/cagri/2019011.

Thiébeau P, Recous S. 2016. Méthode pour quantifier les biomasses de résidus de récolte à la surface des sols après la moisson. Cahiers Agricultures 25: 45001. DOI: 10.1051/cagri/2016027. 
Thiébeau P, Recous S. 2017. Dynamiques de décomposition des résidus de cultures sur des exploitations pratiquant l'agriculture de conservation en région Grand-Est, France. Cahiers Agricultures 26: 65001. DOI: 10.1051/cagri/2017050.

Wilhelm WW, Doran JW, Power JF. 1986. Corn and soybean yield response to crop residue management under no-tillage production systems. Agronomy Journal 78: 184-189. DOI: 10.2134/ agronj1986.00021962007800010036x.

Zhao D, Yang T, An S. 2012. Effects of crop residue cover resulting from tillage practices on LAI estimation of wheat canopies using remote sensing. International Journal of Applied Earth Observation and Geoinformation 14(1): 169-177. DOI: 10.1016/j.jag.2011.09.003.

Citation de l'article : Thiebeau P. 2019. Relation entre taux de couverture du sol et biomasse de résidus de cultures : une simplification prédictive est envisageable. Cah. Agric. 28: 30. 